Georgian Mathematical Journal

Volume 14 (2007), Number 3, 581-595

\title{
GENERALIZED ANALYTIC FUNCTIONS IN HIGHER DIMENSIONS
}

\author{
WOLFGANG TUTSCHKE
}

Dedicated to the memory of I. N. Vekua

on the occasion of his 100th birth anniversary

\begin{abstract}
Originally I. N. Vekua's theory of generalized analytic functions dealt only with linear systems of partial differential equations in the plane. The present paper shows why I. N. Vekua's ideas are also fruitful for the solution of linear and non-linear partial differential equations in higher dimensions.

One of the highlights of the theory of generalized analytic functions in the plane is the reduction of boundary value problems for general (linear or nonlinear) equations to boundary value problems for holomorphic functions using the well-known weakly singular and strongly singular $T$ - and $\Pi$-operators, respectively. The present paper is mainly aimed at reducing boundary value problems in higher dimensions to boundary value problems for monogenic functions.
\end{abstract}

2000 Mathematics Subject Classification: 30G20, 30G35, 35F20, 35F30.

Key words and phrases: Generalized monogenic functions, Clifford-analytic normal form of first order systems, fundamental solution of the CauchyRiemann system of Clifford analysis, reduction of boundary value problems to fixed-point problems.

\section{Goal of the Paper}

Only some of the results of mathematical research have the chance to become a basic component of mathematics. The theory of generalized analytic functions founded by I. N. Vekua [1] and L. Bers [2] succeeded in being included in the pool of important techniques of the theory of partial differential equations. The reason is that the theory of generalized analytic functions is in a position to use the advantages of complex analysis for solving more general systems of partial differential equations than this is possible in the framework of classical complex analysis.

Originally I. N. Vekua's theory investigated only linear uniformly elliptic systems for two desired real-valued functions in the plane. Today I. N. Vekua's ideas are applied to partial differential equations in higher dimensions. Of course, a theory of the same high generality could not yet be developed so far, and in the sequel we shall explain why a comparable generality cannot be expected. 
However, we shall be in a position to reduce boundary value problems for nonlinear systems in higher dimensions to analogous boundary value problems for monogenic functions of Clifford analysis. This method generalizes the reduction of boundary value problems for non-linear systems in the plane to boundary value problems for holomorphic functions.

\section{Short Survey on Generalized Analytic Functions in the Plane}

2.1. Linear systems for two desired real-valued functions. Generalized analytic functions in the sense of I. N. Vekua are solutions to an equation of form

$$
\partial_{\bar{z}} w=A(z) w+B(z) \bar{w} .
$$

This differential equation is the canonical form of a linear and uniformly elliptic first order system of two real equations for two desired real-valued functions $u$ and $v$. The complex rewriting of this system as a complex equation for $w=$ $u+i v$ contains, in general, both first order derivatives $\partial_{z} w$ and $\partial_{\bar{z}} w$. However, carrying out a transformation of the independent variable $z$ and introducing a slightly modified desired function, one comes to the above equation (1). The new independent variable $\zeta$ has to satisfy the Beltrami equation $\partial_{\bar{z}} \zeta=q(z) \partial_{z} \zeta$, where $|q(z)| \leq q_{0}<1$.

The main results of the theory of generalized analytic functions are:

1. Factorization of a generalized analytic function in the form $\Phi w_{0}$ where $\Phi$ is an analytic function and $w_{0} \neq 0$ everywhere.

2. Construction of generalized analytic functions having prescribed zeros and prescribed singularities.

3. Solution of boundary value problems.

The factorization is a special case of a general representation theorem proved by L. Bers and L. Nirenberg [3]. The factorization shows, especially, that the zeros of a generalized analytic function are isolated unless the function vanishes identically (Carleman's Theorem [4]). A short and simple proof of the factorization theorem and of Carleman's Theorem is given by I. N. Vekua [5].

The construction of generalized analytic functions with prescribed zeros leads, in particular, to generalized Cauchy kernels, to a generalized Cauchy Integral Formula and generalized Cauchy type integrals. Boundary value problems for generalized analytic functions can be reduced to singular integral equations for the desired densities of generalized Cauchy type integrals.

2.2. Linear systems for $\mathbf{2} \boldsymbol{n}$ desired real-valued functions. A first generalization of the theory of generalized analytic functions is B. Bojarski's theory [6] of generalized analytic vectors. This is a complex method for the investigation of linear and uniformly elliptic systems of $2 n$ equations for $2 n$ desired realvalued functions $u_{1}, \ldots, u_{n}, v_{1}, \ldots, v_{n}$. Introducing $n$ complex-valued functions $w_{j}=u_{j}+i v_{j}, j=1, \ldots, n$, the system can be reduced to a vector differential equation for $w=\left(w_{1}, \ldots, w_{n}\right)$ of the form

$$
\partial_{\bar{z}} w-Q(z) \partial_{z} w=A(z) w+B(z) \bar{w},
$$


whose coefficients $Q(z), A(z)$ and $B(z)$ are $n \times n$-matrices. Since the complex rewriting of a given system for $2 n$ desired functions contains $2 n$ conplex derivatives $\partial_{z} w_{j}$ and $\partial_{\bar{z}} w_{j}$, in the case $n>1$ it is in general impossible to eliminate the term $\partial_{z} w$ from $(2)$. However, the matrix $Q(z)$ has a special simplified structure.

B. Bojarski's paper [6] contains a general theory. An overview on various types of boundary value problems for generalized analytic vectors can be found in G. F. Manjavidze's and G. Ya. Akhalaya's paper [7].

An alternative approach to complex methods for linear systems of $2 n$ desired real-value functions makes use of hypercomplex numbers $\sum_{k=0}^{r-1}\left(a_{k}+i b_{k}\right) e^{r}$, where $a_{k}$ and $b_{k}$ are real, $i^{2}=-1$, $i e=e i$ and $e^{r}=0$ (see A. Douglis [8]). Using this commutative associative algebra generated by $i$ and $e, \mathrm{R}$. P. Gilbert and G. Hile developed in [9] a generalized hyperanalytic function theory (see also R. P. Gilbert's Lecture Notes [10]).

2.3. Non-linear equations. Provided a certain solvability condition is satisfied (see Subsection 5.2), fully non-linear systems for $2 n$ desired real-valued functions can be reduced to equations of the form

$$
\partial_{\bar{z}} w=F\left(z, w, \partial_{z} w\right)
$$

for the desired vector $w=\left(w_{1}, \ldots, w_{n}\right)$ with complex-valued components (see the paper [11] and the book [12]). While for linear systems in the case $n=1$ the term $\partial_{z} w$ can be eliminated, the normal form (3) for non-linear systems contains this derivative, in general. Nevertheless, boundary value problems for (3) can be reduced to boundary value problems for holomorphic functions. This reduction is based on the following construction:

Consider a non-linear differential equation of the form

$$
L u=F(x, u),
$$

where $x$ varies in a domain $\Omega$ in an Euclidean space. Suppose that $L$ is a linear operator having a fundamental solution $E(x, \xi)$ with singularity at $\xi$. Then solutions of (4) can be represented in the form

$$
u(x)=\tilde{u}(x)+\int_{\Omega} E(x, \xi) F(\xi, u(\xi)) d \xi,
$$

where $\tilde{u}$ is a solution of the linear differential equation $L \tilde{u}=0$. The right-hand side of (5) defines an operator whose fixed points are solutions of (4). Choosing the functions $\tilde{u}$ (depending on $u$ ) in a way such that the right-hand sides of (5) satisfy the given boundary condition, it follows that a possibly existing fixed element is a solution of the boundary value problem under consideration.

Applying this idea to the partial complex differential equation (3), boundary value problems for (3) can be reduced to boundary value problems for holomorphic functions.

Whereas boundary value problems for linear equations can be reduced to (singular) boundary integral equations (see Subsection 2.1), one obtains here domain integral equations. However, this reduction has an important advantage: Since one needs a fundamental solution only to the Cauchy-Riemann 
operator on the left-hand side of (3), the kernel of the corresponding integral equation is always the Cauchy kernel (whereas the Cauchy kernel to a generalized analytic function in the sense of Subsection 2.1 depends on the coefficients $A(z)$ and $B(z)$ of $(1))$. Of course, if the right-hand side $F\left(z, w, \partial_{z} w\right)$ depends not only on $z$ and $w$, but also on $\partial_{z} w$, then there appears the strongly singular $\Pi$-operator (whose kernel is the square of the Cauchy kernel).

A well-posed boundary value problem for (3) is the following Dirichlet problem for a simply connected domain: one prescribes the real part of the desired solution on the whole boundary and the imaginary part at one point (sometimes this problem is quoted as Schwarz problem). This problem is well-posed because the real part of a holomorphic function satisfies the Laplace equation, and thus the real part can be chosen arbitrarily on the whole boundary. After constructing the real part in the whole domain, the imaginary part can be calculated by using the Cauchy-Riemann system. This is a compatible first order system because the real part satisfies the Laplace equation. Consequently, the imaginary part is uniquely determined up to an arbitrary constant.

In case the boundary values of the real part are only continuous, it can happen that the imaginary part does not have limits on the boundary. In order to ensure the existence of the imaginary part in the closure of the (smoothly bounded) domain $\Omega$, one assumes that the boundary values of the real part are Hölder continuous. In case the boundary values of the real part are even Hölder continuously differentiable, the desired solution is Hölder continuously differentiable $\bar{\Omega}$. Then the desired solution turns out to be a fixed-point of the corresponding operator in the space of complex-valued functions which are Hölder continuously differentiable in $\bar{\Omega}$ (see [12]).

In order to solve boundary value problems with boundary data which are only Hölder continuous (but not Hölder continuously differentiable), one can solve the fixed-point problems in the space of Hölder continuous functions whose first order derivatives belong to an $L_{p}$-space. This is done in the paper [13] of G. F. Manjavidze and the author. Still more general boundary data are those which belong piecewise to fractional order spaces. Under this assumption the Dirichlet boundary value problem is solved in the paper [14] of G. F. Manjavidze, H. L. Vasudeva and the author.

\section{Previous Methods and Results in Real Higher-Dimensional EUCLIDEAN SPACES}

Generally speaking, the theory of generalized analytic functions tries to apply methods of complex analysis to systems of differential equations which are more general than the Cauchy-Riemann system. Clearly, the more general a system is, the less general results can be expected. Therefore it makes sense to consider classes of systems which are not too general. In the case of the plane such classes are $p$-analytic and $(p, q)$-analytic functions for which a special theory can be developed (see G. M. Polozhiì's monograph [15]). 
Such relations between the depth of the expected results and the generality of the systems under consideration appear, especially, in the case of higherdimensional (real) Euclidean spaces. Thus understandably the first investigations were devoted to systems with constant coefficients. A. V. Bitsadze [16], for instance, proves the Cauchy-Pompeiu Formula in $\mathbf{R}^{\mathbf{3}}$ implying the Cauchy Integral Theorem for potential vectors $v$ (for which $\operatorname{div} v=\operatorname{curl} v=0$ ). Generalized analytic vectors in higher dimensions can be defined by the generalized Moisil-Théodurescu system

$$
\operatorname{div} v+(a \cdot v)=0, \quad \operatorname{grad} \varphi+\operatorname{curl} v+[v \times b]+c \varphi=0
$$

and the generalized Riesz system

$$
\sum\left[\partial_{x_{j}} u_{j}+a_{j} u_{j}\right]=0, \quad \partial_{x_{k}} u_{j}-\partial_{x_{j}} u_{k}-b_{k} u_{j}+b_{j} u_{k}=0,
$$

respectively. A unified approach to such systems is possible in the framework of Clifford analysis because both systems are special cases of $D u+H u=0$, where $u$ is the desired Clifford-algebra-valued function and $D=\sum_{j=0}^{n} e_{j} \partial_{x_{j}}$ is the Cauchy-Riemann operator of Clifford analysis, where, as usual, $e_{0}=1$, $e_{1}^{2}=\cdots=e_{n}^{2}=-1$ and $e_{j} e_{k}+e_{k} e_{j}=0$ if $j, k=1, \ldots, n$ and $j \neq k$. If, in particular, the coefficient $H$ is a constant (in the Clifford algebra), the above linear differential equation for generalized analytic functions has been investigated by E. Obolashvili (see her book [17]; if $H$ is a constant, then solutions can be constructed using Fourier integral transformations). Higher order differential equations can also be solved in the framework of Clifford analysis, see K. Gürlebeck and W. Sprößig [18], V. V. Kravchenko and M. V. Shapiro [19] and E. Obolashvili [20]. Assuming that $e_{j}^{2}=+1$ or $e_{j}^{2}=0$ for some $j$, hyperbolic and parabolic differential equations can also be included in Clifford analysis.

Solving real partial differential equations within the framework of Clifford analysis has, generally speaking, some advantages. Firstly, it leads to the unification of statements, secondly, in some cases one obtains simpler explicit representations. However, using the matrix notation instead of a rewriting in the language of Clifford analysis, sometimes more general results can be obtained. An example is given in the paper [21], where an initial value problem for a hyperbolic first order system is solved using the matrix notation. The solvability conditions obtained in [21] are weaker than those obtained by E. Obolashvili in [17] in the framework of Clifford analysis.

\section{Boundary Value Problems for Monogenic Functions}

A (left-)monogenic function is a solution of the differential equation $D u=0$. Its real-valued components are solutions of the Laplace equation. However, they are not independent of each other because they are connected by the CauchyRiemann system $D u=0$. In other words, one cannot arbitrarily prescribe the boundary values of all of the real-valued components. The situation is similar to the case of holomorphic functions in the complex plane: Prescibing the real

part on the boundary, the imaginary part is then already uniquely determined 
up to an arbitrary constant (see Subsection 2.3). In the sequel we shall discuss the case of $\mathbf{R}^{\mathbf{3}}$.

Denote the independent variables in $\mathbf{R}^{\mathbf{3}}$ by $x_{0}, x_{1}, x_{2}$. The real-valued components of a monogenic functions are denoted by $u_{0}, u_{1}, u_{2}, u_{12}$. Then the Cauchy-Riemann system can be written in the form

$$
\begin{aligned}
& \partial_{x_{0}} u_{0}=\partial_{x_{1}} u_{1}+\partial_{x_{2}} u_{2}, \\
& \partial_{x_{0}} u_{12}=-\partial_{x_{1}} u_{2}+\partial_{x_{2}} u_{1}, \\
& \partial_{x_{1}} u_{12}=+\partial_{x_{2}} u_{0}+\partial_{x_{0}} u_{2}, \\
& \partial_{x_{2}} u_{12}=-\partial_{x_{0}} u_{1}-\partial_{x_{1}} u_{0} .
\end{aligned}
$$

In order to formulate a well-posed boundary value problem, we consider cylindrical domains $\Omega$ of the form

$$
\Omega=\left\{x=\left(x_{0}, x_{1}, x_{2}\right): \psi_{1}\left(x_{1}, x_{2}\right) \leq x_{0} \leq \psi_{2}\left(x_{1}, x_{2}\right),\left(x_{1}, x_{2}\right) \in M\right\},
$$

where $M$ is a simply connected domain in the $\left(x_{1}, x_{2}\right)$-plane and $\psi_{1}$ and $\psi_{2}$ are continuously differentiable in $\bar{M}$. Then the lower base of $\bar{\Omega}$ in the direction of the $x_{0}$-axis is

$$
S=\left\{x=\left(x_{0}, x_{1}, x_{2}\right): x_{0}=\psi_{1}\left(x_{1}, x_{2}\right),\left(x_{1}, x_{2}\right) \in M\right\} .
$$

Since the real-valued components of a monogenic function are solutions of the Laplace equation, the following Dirichet boundary value problem is uniquely solvable for the Cauchy-Riemann system (6)-(9):

Find a monogenic function $u$ satisfying the following conditions:

- Two components $u_{1}$ and $u_{2}$ are arbitrarily prescribed on the whole boundary $\partial \Omega$ of $\Omega$.

- The component $u_{0}$ is given on the lower base $S$.

- The last component $u_{12}$ can be chosen arbitrarily at one point of $\Omega$.

Indeed, after having chosen $u_{1}$ and $u_{2}$, the right-hand side of (6) is a given continuous function (provided the boundary values of $u_{1}$ and $u_{2}$ are continuously differentiable on $S$ ). Then equation (6) leads for each fixed pair $\left(x_{1}, x_{2}\right)$ to $u_{0}\left(x_{0}, x_{1}, x_{2}\right)$ by integration in the $x_{0}$-direction.

It remains to calculate $u_{12}$. Equations (7), (8) and (9) are a first order system of the form

$$
\partial_{x_{j}} u_{12}=p_{j}, \quad j=0,1,2
$$

whose right-hand sides are now given functions depending on $x$. The right-hand sides are compatible, because we have, for instance,

$$
\begin{aligned}
\partial_{x_{1}} p_{0}-\partial_{x_{0}} p_{1} & =\left(-\partial_{x_{1}}^{2} u_{2}+\partial_{x_{1}} \partial_{x_{2}} u_{1}\right)-\left(\partial_{x_{0}} \partial_{x_{2}} u_{0}+\partial_{x_{0}}^{2} u_{2}\right) \\
& =\partial_{x_{2}}^{2} u_{2}+\partial_{x_{1}} \partial_{x_{2}} u_{1}-\partial_{x_{0}} \partial_{x_{2}} u_{0} \\
& =\partial_{x_{2}}\left(\partial_{x_{2}} u_{2}+\partial_{x_{1}} u_{1}-\partial_{x_{0}} u_{0}\right)=0
\end{aligned}
$$

in view of the Laplace equation for $u_{2}$ and equation (6).

In the literature one can also find other boundary conditions. E. Obolashvili [22] prescribes, for instance, two components on the whole boundary, whereas 
the two remaining coefficients have to satisfy the Riemann-Hilbert boundary condition on a curve on the boundary. The above-formulated boundary condition is, however, an immediate generalization of the boundary condition for holomorphic functions discussed in Subsection 2.3.

\section{Generalized Monogenic Functions}

5.1. Definition. A generalized analytic function in the $z$-plane is a function $w(z)$ in case $\partial_{\bar{z}} w$ can be expressed by $z, w$ and $\partial_{z} w$ (see equation (3)). Since Clifford analysis replaces the Cauchy-Riemann equation $\partial_{\bar{z}} w=0$ by $D u=0$, a generalized monogenic function can be defined as a solution of a differential equation of the form

$$
D u=F\left(x, u, \partial_{x_{0}} u_{A}, \ldots, \partial_{x_{n}} u_{A}\right),
$$

where $x=\left(x_{0}, x_{1}, \ldots, x_{n}\right)$ and $u_{A}, A \in\{0,1, \ldots, n, 12, \ldots, 12 \ldots n\}$, are the $2^{n}$ real-valued components of $u$. Of course, the differential equation is a linear one if its right-hand side $F$ is linear in $u_{A}$ and $\partial_{x_{j}} u_{A}, j=0,1, \ldots, n$. Using the Beltrami equation in the case of a scalar-valued linear differential equation in the plane, the term $\partial_{z} w$ on the right-hand side can be eliminated (see Subsection 2.1). This elimination is no longer possible already in the case of generalized analytic vectors so that the corresponding canonical form (2) of the system under consideration contains the term $\partial_{z} w$, in general. The same situation appears in the case of linear systems (10) because one has only $n+1$ coordinates, whereas the number of (real) derivatives equals $(n+1) 2^{n}$. Consequently, linear equations of the form $D u=F(x, u)$ (where the right-hand side does not depend on the derivatives) are only special systems. Nevertheless, for such linear equations a far-reaching theory of qualitative properties of solutions of such systems is developed in B. Goldschmidt's papers [23, 24].

On the one hand, also in the case of linear systems (10) one cannot eliminate all first order derivatives on the right-hand side, as this is possible in the case of scalar linear equations (1) in the plane. On the other hand, we shall show that all $2^{n}$ first order derivatives $\partial_{x_{0}} u_{A}$ can be eliminated. This can be done even in the case of fully non-linear systems (see Subsection 5.3 below). This construction generalizes an analogous procedure for fully non-linear systems in the plane which will be sketched in the following Subsection 5.2.

5.2. A complex normal form for first order systems in the plane. A fully non-linear first order system for two desired real-valued functions $u(x, y)$ and $v(x, y)$ has the form

$$
H_{j}\left(x, y, u, v, \partial_{x} u, \partial_{y} u, \partial_{x} v, \partial_{y} v\right)=0, \quad j=1,2 .
$$

Introduce four linear combinations $p_{1}, p_{2}, q_{1}, q_{2}$ of the first order derivatives $\partial_{x} u, \partial_{y} u, \partial_{x} v, \partial_{y} v$, where $p_{1}+i p_{2}=\partial_{z}(u+i v)$ and $q_{1}+i q_{2}=\partial_{\bar{z}}(u+i v)$. Then replace the first order derivatives of $u$ and $v$ in (11) by these linear combinations. In case the new system can be solved with respect to $q_{1}$ and $q_{2}$, one obtains the complex normal form (3), cf. [11, 12].

Next an analogous construction will be carried out in higher dimensions. 


\subsection{A Clifford-analytic normal form of first order systems in higher} dimensions. Consider a fully non-linear first order system of $2^{n}$ equations for $2^{n}$ desired real-valued functions $u_{0}, u_{1}, \ldots, u_{n}, u_{12}, \ldots, u_{12 \ldots n}$ depending on $n+1$ real variables $x_{0}, x_{1}, \ldots, x_{n}$ :

$$
H_{j}\left(x_{0}, \ldots, x_{n}, u_{0}, \ldots, u_{12 \ldots n}, \partial_{x_{0}} u_{0}, \ldots, \partial_{x_{n}} u_{12 \ldots n}\right)=0, \quad j=1, \ldots, 2^{n} .
$$

The $2^{n}$ desired real-valued functions can be interpreted as the components of a Clifford-algebra-valued function $u(x)$ depending on $x=\left(x_{0}, x_{1}, \ldots, x_{n}\right)$. Clearly, $D u$ has also $2^{n}$ real components $q_{A}$ which can be expressed by the first order derivatives of the real-valued components $u_{A}$ of $u$. The definition of the Cauchy-Riemann operator $D$ shows that

$$
q_{A}=\partial_{x_{0}} u_{A}+\text { derivatives with respect to } x_{1}, \ldots, x_{n} .
$$

Consequently, all derivatives with respect to $x_{0}$ can be expressed by the $2^{n}$ real variables $q_{A}$ and derivatives with respect to $x_{1}, \ldots, x_{n}$. Carrying out this substitution, system (12) turns out to be a system of $2^{n}$ equations depending on the $(n+1)+(n+2) 2^{n}$ variables $x_{0}, \ldots, x_{n}, u_{A}, q_{A}, \partial_{x_{k}} u_{A}, k=1, \ldots, n$. Now assume that this system can be solved for the $2^{n}$ variables $q_{A}$. Then system (12) passes into its Clifford-analytic normal form

$$
D u=F\left(x, u, \partial_{x_{1}} u_{0}, \ldots, \partial_{x_{n}} u_{12 \ldots n}\right) .
$$

5.4. Reduction of boundary value problems to fixed-point problems. Consider a first order system, for instance system (12) in its Clifford-analytic normal form (13). Note that

$$
\frac{1}{\omega_{n+1}} \cdot \frac{\bar{x}-\bar{\xi}}{|x-\xi|^{n+1}}
$$

is a fundamental solution of the Cauchy-Riemann equation $D u=0$ in $\mathbf{R}^{\mathbf{n}+\mathbf{1}}$ with singularity at $\xi$ (where $\omega_{n+1}$ is the surface measure of the unit sphere, while $\bar{x}$ means $x_{0}-e_{1} x_{1}-\cdots-e_{n} x_{n}$ in case $\left.x=x_{0}+e_{1} x_{1}+\cdots=e_{n} x_{n}\right)$. By representation (5) in Subsection 2.3, a solution $u(x)$ of (13) in $\Omega$ can be represented by

$$
u(x)=\tilde{u}(x)+\frac{1}{\omega_{n+1}} \int_{\Omega} \frac{\bar{x}-\bar{\xi}}{|x-\xi|^{n+1}} F\left(\xi, u(\xi), \partial_{x_{1}} u_{A}(\xi), \ldots, \partial_{x_{n}} u_{A}(\xi)\right) d \xi,
$$

where $\tilde{u}$ is monogenic. Therefore fixed points of the operator

$$
U(x)=\tilde{u}(x)+\frac{1}{\omega_{n+1}} \int_{\Omega} \frac{\bar{x}-\bar{\xi}}{|x-\xi|^{n+1}} F\left(\xi, u(\xi), \partial_{x_{1}} u_{A}(\xi), \ldots, \partial_{x_{n}} u_{A}(\xi)\right) d \xi
$$

are solutions of the non-linear equation (13) provided $\tilde{u}$ is monogenic.

Now we consider the boundary value problem

$$
\mathcal{B} u=g \text {. }
$$

Suppose that this boundary value problem is uniquely solvable for monogenic functions. Then one gets the following statement. 
Theorem. Choosing the monogenic function $\tilde{u}$ depending on $u$ as a solution of the boundary value problem

$$
\mathcal{B} \tilde{u}(x)=g(x)-\frac{1}{\omega_{n+1}} \int_{\Omega} \frac{\bar{x}-\bar{\xi}}{|x-\xi|^{n+1}} F\left(\xi, u(\xi), \partial_{x_{1}} u_{A}(\xi), \ldots, \partial_{x_{n}} u_{A}(\xi)\right) d \xi,
$$

the fixed points of operator (15) are solutions of the boundary value problem (13), (16).

The existence of fixed points can be proved using fixed-point theorems such as the contraction-mapping principle. Notice, finally, that this reduction of the boundary value problem (13), (16) to the fixed-point problem for the operator (15) is also possible if the right-hand side of (13) depends on the derivatives $\partial_{x_{0}} u_{A}$. In such a case the reduction of this system to the Clifford-analytic normal form (13) is not necessary.

5.5. Application of fixed-point theorems. The investigation of operator (15) in the framework of Clifford analysis is similar as this can be done in the complex plane (see Subsection 2.3). In the sequel we sketch the crucial points. Calculating the derivatives of the images $U(x)$ with respect to $x_{j}, j=0,1, \ldots, n$, the following strongly singular integrals occur

$$
\int_{\Omega} h(\xi) \partial_{x_{j}}\left(\frac{\bar{x}-\bar{\xi}}{|x-\xi|^{n+1}}\right) d \xi
$$

where $h(\xi)=F\left(\xi, u(\xi), \partial_{x_{1}} u_{A}(\xi), \ldots, \partial_{x_{n}} u_{A}(\xi)\right)$. This can be rewritten as

$$
\int_{\Omega}(h(\xi)-h(x)) \partial_{x_{j}}\left(\frac{\bar{x}-\bar{\xi}}{|x-\xi|^{n+1}}\right) d \xi+h(x) \int_{\Omega} \partial_{x_{j}}\left(\frac{\bar{x}-\bar{\xi}}{|x-\xi|^{n+1}}\right) d \xi .
$$

Suppose the right-hand side $F$ satisfies a Lipschitz condition with respect to the desired $u_{A}$ and their derivatives. Then the absolute value of $h(\xi)-h(x)$ can be estimated by const $\cdot|x-\xi|^{\lambda}$ provided $u$ is Hölder continuously differentiable with exponent $\lambda, 0<\lambda<1$. Thus the first integral in (18) turns out to be weakly singular.

Let $\Omega_{\varepsilon}$ be the domain $\Omega$ from which the ball $|x-\xi| \leq \varepsilon$ is removed. Then

$$
\int_{\Omega_{\varepsilon}} \partial_{x_{j}}\left(\frac{\bar{x}-\bar{\xi}}{|x-\xi|^{n+1}}\right) d \xi=\int_{\partial \Omega} \frac{\bar{x}-\bar{\xi}}{|x-\xi|^{n+1}} N_{j} d \mu+\int_{|x-\xi|=\varepsilon} \frac{\bar{x}-\bar{\xi}}{|x-\xi|^{n+1}} N_{j} d \mu,
$$

where $\left(N_{0}, N_{1}, \ldots, N_{n}\right)$ is the outer unit normal and $d \mu$ is the measure element on the boundary. Notice that the Euclidean length of $\bar{x}-\bar{\xi}$ equals $\varepsilon$ and $d \mu=\varepsilon^{n} d \mu_{1}$, where $d \mu_{1}$ is the surface element of the unit sphere in $\mathbf{R}^{\mathbf{n}+\mathbf{1}}$. Hence the second integral on the right-hand side of the last formula does not depend on $\varepsilon$, and so its limit exists as $\varepsilon \rightarrow 0$. Consequently, the second integral in (18) exists as a principal value, and it can be expressed by a Cauchy type integral over $\partial \Omega$. 
Mapping properties of singular integral operators can be found, for instance, in C. Miranda's book [25]. Concerning the application of fixed-point methods to partial differential equations see also [26].

\section{Recent Trends. Outlook}

6.1. Specific features of higher-dimensional cases. On the one hand, the preceding sections show that many constructions of the theory of generalized analytic functions in the complex plane can be carried out in higher-dimensional Euclidean spaces as well. On the other hand, in the higher-dimensional case many aspects are still to be developed in greater detail. This concerns, for instance, boundary value problems for monogenic functions, Cauchy type integrals, singular integral equations in higher dimensions, and mapping properties of integral operators.

6.2. Generalized analytic functions in several complex variables. Generalizations of the theory of generalized analytic functions considered in the present paper concern generalizations to real higher-dimensional spaces. However, there are also generalizations to several complex variables. In the paper [27], for instance, I. N. Vekua's idea [5] for proving Carleman's Theorem (see Subsection 2.1) has been used in order to prove a factoriation theorem for generalized analytic functions in several complex variables. Under suitable assumptions one can show that the set of all zeros of a generalized analytic function in several complex variables is an analytic set (in the sense of the theory of holomorphic functions in several complex variables), whereas the zeros are isolated in the case of a not identically vanishing generalized analytic function in the complex plane.

Many interesting results have also been obtained for second order elliptic equations in several complex variables, see H. Begehr's and A. Dzhuraev's article [28], where further references can be found. Such systems generalize, in particular, the famous Bitsadze equation $\partial_{\bar{z}}{ }^{2} u=0$ (cf. [29]) which is elliptic but not strongly elliptic.

6.3. Generalized analytic and generalized monogenic functions as initial function. Most of the applications of complex methods to partial differential equations deal with boundary value problems. Initial value problems, however, can also be solved using tools of complex analysis. Cauchy-Kovalevskaya problems, for instance, are solvable in case the initial functions are holomorphic. Since holomorphic and generalized analytic functions have many common properties, one can expect that initial value problems with generalized analytic initial functions are also solvable. This is indeed possible, see the paper [30] or the booklet [31]. An important tool for the corresponding constructions is again the representation of generalized analytic functions by holomorphic ones.

Concerning higher dimensions, one can also solve initial value problems with monogenic or generalized monogenic functions. A special class of generalized 
monogenic functions is the one satisfying a differential equation with the antimonogenic right-hand side (in the case of the complex plane a differential equation with an anti-monogenic right hand side has the form $\left.\partial_{\bar{z}} w=b(z) \bar{w}\right)$. Further references can be found in the paper [32] of Nguyen Thanh Van and the author.

The possibility to solve initial value problems in suitably chosen classes of generalized analytic or generalized monogenic functions leads, finally, to the concept of associated spaces (see [33]): an initial function belonging to an associated space is admissible. Since an evolution operator may possess different associated spaces, one can decompose initial functions into components belonging to different associated spaces. That way even ill-posed initial value problems are solvable (see [34]).

6.4. The Cimmino system. At present, complex methods for partial differential equations in higher dimensions are mainly applied either in the framework of Clifford-algebra-valued functions or in the framework of (complex-valued) holomorphic functions in several complex variables. In both cases the number of desired (real-valued) functions is different from the number of (real-valued) independent variables. That makes a big difference from the case of complexvalued functions in the plane, where both numbers of (real) independent and dependent variables equal two.

There is another possibility to apply complex methods to systems of partial differential equations with the same number of (more than two) independent and dependent variables. This is the case of the Cimmino system

$$
\begin{gathered}
\partial_{x_{1}} u_{1}-\partial_{x_{2}} u_{2}+\partial_{x_{3}} u_{3}-\partial_{x_{4}} u_{4}=0, \\
\partial_{x_{2}} u_{1}+\partial_{x_{1}} u_{2}-\partial_{x_{4}} u_{3}-\partial_{x_{3}} u_{4}=0, \\
\partial_{x_{3}} u_{1}-\partial_{x_{4}} u_{2}-\partial_{x_{1}} u_{3}+\partial_{x_{2}} u_{4}=0 \\
\partial_{x_{4}} u_{1}+\partial_{x_{3}} u_{2}+\partial_{x_{2}} u_{3}+\partial_{x_{1}} u_{4}=0
\end{gathered}
$$

for four desired real-valued functions $u_{j}$ depending on four real variables $x_{j}$, $j=1,2,3,4$. This system was introduced in 1941 by G. Cimmino [35] in connection with systems whose solutions satisfy the Laplace equation. Using its complex form $\partial_{\bar{z}} f+\partial_{w} \bar{g}=0, \partial_{\bar{w}} f-\partial_{z} \bar{g}=0$ (where $z=x_{1}+i x_{2}, w=x_{3}+i x_{4}$, $\left.f=u_{1}+i u_{2}, g=u_{3}+i u_{4}\right)$, recently Sorin Dragomir and E. Lanconelli [36] resumed investigating the Cimmino system. They proved, for instance, the Cauchy-Pompeiu Integral Formula.

The Cimmino system (19)-(22) is a system in four real variables, whereas the Cauchy-Riemann system (6)-(9) is a system in only three real variables. Therefore the Dirichlet boundary value problem of Section 4 should to be modified, and we shall come to an easier formulation. Indeed, first one has to take into consideration that all components $u_{j}$ of a solution to the Cimmino system satisfy the Laplace equation. Here even three components $u_{1}, u_{2}, u_{3}$ can be arbitrarily prescribed on the whole boundary. Then the Cimmino system can be interpreted as a first order system of the form $\partial_{x_{j}} u_{4}=p_{j}, j=1,2,3,4$, for the fourth component $u_{4}$. One has $p_{1}=-\partial_{x_{4}} u_{1}-\partial_{x_{3}} u_{2}-\partial_{x_{2}} u_{3}, p_{2}=-\partial_{x_{3}} u_{1}+\partial_{x_{4}} u_{2}+\partial_{x_{1}} u_{3}$ and so on. A straightforward calculation shows that this system is compatible 
because one has, for instance, $\partial_{x_{2}} p_{1}-\partial_{x_{1}} p_{2}=0$ in view of the Laplace equation for $u_{3}$ and equations (19) and (20). Consequently, $u_{4}$ can be arbitrarily prescribed at one point (we suppose, of course, that each closed curve is homotopically equal to a point).

So far the inhomogeneous Cimmino system has been investigated only for right-hand sides depending only on the variable $x=\left(x_{1}, x_{2}, x_{3}, x_{4}\right)$. The question is, how general Cimmino systems are whose right-hand sides depend on the desired $u=\left(u_{1}, u_{2}, u_{3}, u_{4}\right)$ and its first order derivatives. Consider a first order system

$$
H_{j}\left(x, u, \partial_{x_{1}} u_{1}, \ldots, \partial_{x_{4}} u_{4}\right)=0, \quad j=1,2,3,4 .
$$

Denote the four linear combinations of the first order derivatives on the lefthand side of (19)-(22) by $q_{1}, q_{2}, q_{3}$ and $q_{4}$ respectively. So we have, for instance, $\partial_{x_{1}} u_{1}-\partial_{x_{2}} u_{2}+\partial_{x_{3}} u_{3}-\partial_{x_{4}} u_{4}=q_{1}$. Clearly, the four derivatives $\partial_{x_{4}} u_{j}$ can be expressed by $q_{1}, q_{2}, q_{3}, q_{4}$. Substituting these expressions into (23), one obtains a system of four equations in $x, u, q_{1}, q_{2}, q_{3}, q_{4}, \partial_{x_{1}} u_{1}, \ldots, \partial_{x_{3}} u_{4}$. Now suppose that the new system can be solved for $q_{1}, q_{2}, q_{3}, q_{4}$. Then (23) takes the form

$$
\begin{aligned}
& \partial_{x_{1}} u_{1}-\partial_{x_{2}} u_{2}+\partial_{x_{3}} u_{3}-\partial_{x_{4}} u_{4}=F_{1}\left(x, u, \partial_{x_{1}} u_{1}, \ldots, \partial_{x_{3}} u_{4}\right), \\
& \partial_{x_{2}} u_{1}+\partial_{x_{1}} u_{2}-\partial_{x_{4}} u_{3}-\partial_{x_{3}} u_{4}=F_{2}\left(x, u, \partial_{x_{1}} u_{1}, \ldots, \partial_{x_{3}} u_{4}\right), \\
& \partial_{x_{3}} u_{1}-\partial_{x_{4}} u_{2}-\partial_{x_{1}} u_{3}+\partial_{x_{2}} u_{4}=F_{3}\left(x, u, \partial_{x_{1}} u_{1}, \ldots, \partial_{x_{3}} u_{4}\right), \\
& \partial_{x_{4}} u_{1}+\partial_{x_{3}} u_{2}+\partial_{x_{2}} u_{3}+\partial_{x_{1}} u_{4}=F_{4}\left(x, u, \partial_{x_{1}} u_{1}, \ldots, \partial_{x_{3}} u_{4}\right) .
\end{aligned}
$$

The new system can be considered as some kind of normal form which is similar to the normal forms (3) and (13) in Subsections 2.3 and 5.3, respectively.

6.5. Quaternionic analysis. Generally speaking, generalized monogenic functions in Clifford analysis are solutions of systems of $2^{n}$ real equations for $2^{n}$ desired real-valued functions in $n+1$ real variables. The numbers of desired functions and independent real variables coincide only in the case of the complex plane $(n=1)$. However there is still another case in the framework of Clifford analysis in which the number of desired real-valued components is equal to the number of real variables. This is the case of quaternionic analysis.

Quaternions $x=x_{1}+i x_{2}+j x_{3}+k x_{4}$ have four real components $x_{1}, x_{2}, x_{3}, x_{4}$, where $i^{2}=j^{2}=k^{2}=-1$ and $i j=k, j k=i, k i=j$. Consider a quaternionvalued function $u=u_{1}+i u_{2}+j u_{3}+k u_{4}$ depending on a quaternion-valued variable $x$. Then the so-called (left-)regular functions of quaternionic analysis are defined by $D u=0$, where $D$ is the Cauchy-Riemann operator $D=\partial_{x_{1}}+$ $i \partial_{x_{2}}+j \partial_{x_{3}}+k \partial_{x_{4}}$. Using the quaternionic variable $x=x_{1}+i x_{2}-j x_{3}+k x_{4}$, the component-wise rewriting of the Cauchy-Riemann equation $D u=0$ is identical with the Cimmino system (19)-(22). Therefore the (non-linear) generalized Cimmino system (24)-(27) can be solved using tools of Clifford analysis. For instance, with the help of the Cauchy kernel (14) a boundary value problem for the non-linear system (24)-(27) can be reduced to an analogous boundary value problem for the Cimmino system. The corresponding fixed-point problem can be solved by the contraction-mapping principle in case the right-hand side satisfies a Lipschitz condition with Lipschitz constants which are small enough. 
The investigation of generalized inhomogeneous Cimmino systems with an arbitrary number of desired real-valued functions and with the same number of independent real variables could lead to an alternative to generalized monogenic functions. It seems to be clear that the ideas of the theory of generalized analytic functions will also be useful for solving such generalized Cimmino systems.

\section{REFERENCES}

1. I. N. VekUa, Generalized analytic functions. 2nd ed. (Russian) Nauka, Moscow, 1988; English transl.: Pergamon Press, London-Paris-Frankfurt; Addison-Wesley Publishing Co., Inc., Reading, Mass. 1962.

2. L. Bers, Theory Theory of pseudo-analytic functions. Institute for Mathematics and Mechanics, New York University, New York, 1953.

3. L. Bers and L. NirenberG, On a representation theorem for linear elliptic systems with discontinuous coefficients and its applications. Convegno Internazionale sulle Equazioni Lineari alle Derivate Parziali, Trieste, 1954, pp. 111-140. Edizioni Cremonese, Roma, 1955.

4. T. Carleman, Sur les systémes linéaires aux dérivées partielles du premier ordre à deux variables. C. R. Paris 197(1933), 471-474.

5. I. N. VEKUA, On a property of solutions of a generalized system of Cauchy-Riemann equations. (Russian) Soobshch. Akad. Nauk Gruzin. SSR 14(1953), 449-453.

6. B. V. Bojarskil̆, Theory of generalized analytic vectors. (Russian) Ann. Polon. Math. 17(1966), 281-320.

7. G. Manjavidze and G. Akhalaia, Boundary value problems of the theory of generalized analytic vectors. Complex methods for partial differential equations (Ankara, 1998), 57-95, Int. Soc. Anal. Appl. Comput., 6, Kluwer Acad. Publ., Dordrecht, 1999.

8. A. Douglis, A function-theoretic approach to elliptic systems of equations in two variables. Comm. Pure Appl. Math. 6(1953), 259-289.

9. R. P. Gilbert and G. N. Hile, Generalized hyperanalytic function theory. Bull. Amer. Math. Soc. 78(1972), 998-1001.

10. R. P. Gilbert, Constructive methods for elliptic equations. Lecture Notes in Mathematics, Vol. 365. Springer-Verlag, Berlin-New York, 1974.

11. W. Tutschke, Solution of non-linear first order systems of partial differential equations using a complex normal form. (German) Math. Nachr. 75(1976), 283-298.

12. W. Tutschke, Partial differential equations. Classical, functional-analytical and complex methods. (German) Teubner-Texte zur Mathematik [Teubner Texts in Mathematics], 27. BSB B. G. Teubner Verlagsgesellschaft, Leipzig, 1983.

13. G. F. Manjavidze and W. Tutschke, Some boundary value problems for first-order nonlinear differential systems on the plane. (Russian) Boundary value problems of the theory of generalized analytic functions and their applications, 79-124, Tbilis. Gos. Univ., Tbilisi, 1983.

14. G. F. Manjavidze, W. Tutschke, and H. L. Vasudeva, A complex method for solving boundary value problems with boundary data piecewise belonging to fractional order spaces. Mem. Differential Equations Math. Phys. 33(2004), 103-120.

15. G. M. PolozhII, The theory and application of $p$-analytic and and $(p, q)$-analytic functions. Generalizations of the theory of analytic functions of a complex variable. 2nd ed. (Russian) Naukova Dumka, Kiev, 1973.

16. A. V. Bitsadze, Spatial analogue of an integral of Cauchy type and some of its applications. (Russian) Izvestiya Akad. Nauk SSSR. Ser. Mat. 17(1953), 525-538. 
17. E. Oвоlashvili, Partial differential equations in Clifford analysis. Pitman Monographs and Surveys in Pure and Applied Mathematics, 96. Longman, Harlow, 1998.

18. K. GürLEBECK and W. Sprössig, Quaternionic analysis and elliptic boundary value problems. Mathematical Research, 56. Akademie-Verlag, Berlin, 1989. Quaternionic analysis and elliptic boundary value problems. International Series of Numerical Mathematics, 89. Birkhäuser Verlag, Basel, 1990.

19. V. V. Kravchenko and M. V. Shapiro, Integral representations for spatial models of mathematical physics. Pitman Research Notes in Mathematics Series, 351. Longman, Harlow, 1996.

20. E. OBolashvili, Higher order partial differential equations in Clifford analysis. Effective solutions to problems. Progress in Mathematical Physics, 28. Birkhäuser Boston, Inc., Boston, MA, 2003.

21. W. Tutschke, Solution of initial value problems for first order systems using matrix notation. Begehr special issue. Appl. Anal. 73(1999), No. 1-2, 271-280.

22. Е. Овоlashvili, Effective solutions of some boundary value problems in two- and threedimensional cases. Functional analytic methods in complex analysis and applications to partial differential equations (Trieste, 1988), 149-172, World Sci. Publ., River Edge, NJ, 1990.

23. B. GoldSchmidt, Existence and representation of solutions of a class of elliptic systems of partial differential equations of first order in the space. Math. Nachr. 108(1982), 159166.

24. B. Goldschmidt, A Cauchy integral formula for a class of elliptic systems of partial differential equations of first order in the space. Math. Nachr. 108(1982), 167-178.

25. C. Miranda, Partial differential equations of elliptic type. Second revised edition. (Translated from the Italian) Ergebnisse der Mathematik und ihrer Grenzgebiete, Band 2. Springer-Verlag, New York-Berlin, 1970.

26. W. Tutschke and C. J. VAnEgas, Fixed-point theorems and applications to differential equations. XVIII Escuela Venezolana de Matemáticas, Mérida (Venezuela), September 49, 2005.

27. W. Tutschke, A new application of I. N. Vekua's proof for Carleman's theorem. (Russian) Dokl. Akad. Nauk SSSR 214(1974), 1276-1279; English transl.: Soviet Math. Dokl. 15(1974), 374-378.

28. H. Begehr and A. Dzhuraev, Overdetermined systems of second order elliptic equations in several complex variables. Generalized analytic functions (Graz, 1997), 89-109, Int. Soc. Anal. Appl. Comput., 1, Kluwer Acad. Publ., Dordrecht, 1998.

29. A. V. Bitsadze, On the uniqueness of the solution of the Dirichlet problem for elliptic partial differential equations. (Russian) Uspehi Matem. Nauk (N.S.) 3(1948), No. 6(28), 211-212.

30. W. Tutschke, A problem with initial values for generalized analytic functions depending on time (generalizations of the Cauchy-Kowalewski and Holmgren theorems) (Russian). Dokl. Akad. Nauk USSR 262(1982), No. 5, 1081-1085; English transl.: Soviet Math. Dokl. 25(1982), No. 1, 201-205.

31. W. Tutschke, Solution of initial value problems in classes of generalized analytic functions. The method of scales of Banach spaces. Teubner-Texte zur Mathematik [Teubner Texts in Mathematics], 110. BSB B. G. Teubner Verlagsgesellschaft, Leipzig, 1989; Springer-Verlag, Berlin, 1989.

32. W. Tutschke and Nguyen Thanh Van, Interior estimates in the sup-norm for generalized monogenic functions satisfying a differential equation with an anti-monogenic right-hand side. Complex Variables and Elliptic Equations 52 (2007), No. 5, 367-375. 
33. W. Tutschke, Associated spaces - a new tool of real and complex analysis (in preparation).

34. W. Tutschke, Associated partial differential operators - applications to well- and illposed problems. Abstract and applied analysis, 373-383, World Sci. Publ., River Edge, NJ, 2004.

35. G. Cimmino, Su alcuni sistemi lineari omogenei di equazioni alle derivate parziali del primo ordine. (Italian) Rend. Sem. Mat. Univ. Padova 12(1941), 89-113.

36. S. Dragomir and E. LAnconelli, On first order linear PDE systems all of whose solutions are harmonic functions. Tsukuba J. Math. 30(2006), No. 1, 149-170.

(Received 14.03.2007)

Author's address:

Graz University of Technology

Department of Numerical Mathematics

Steyrergasse 30/3, A-8010 Graz

Austria

E-mail: tutschke@tugraz.at 